\title{
Effects of boundary condition in numerical simulations of vortex dynamics
}

\author{
By D. S. PRADEEP AND F. HUSSAIN \\ Department of Mechanical Engineering, University of Houston, Houston, TX 77204-4006, USA
}

(Received 8 May 2004 and in revised form 6 July 2004)

\begin{abstract}
We show that the periodic boundary conditions typically applied in numerical simulations of vortex dynamics can lead to significantly incorrect results even when the vortex cores are small compared to the computational domain. This is demonstrated for two previously studied flows which capture significant flow physics: (i) an isolated vortex embedded in fine-scale turbulence; (ii) two antiparallel vortices of unequal strength undergoing reconnection. In case (i), periodicity, when invoked, results in strong, unphysical turbulence growth leading to vortex core transition, whereas the vortex remains totally intact during its interaction with the turbulence when periodicity is not invoked. In case (ii), the vortex interaction, including reconnection, is significantly distorted. These differences are due to the artificial zero circulation constraint, inherent in periodic simulations.
\end{abstract}

The Fourier pseudospectral algorithm with triply periodic boundary conditions (TP) (Canuto et al. 1988) is commonly used in direct numerical simulations (DNS) of nominally unbounded flows (i.e. flows away from walls). In addition to its application to homogeneous turbulence, the method has been extensively used to study vortex dynamics such as pairing and reconnection, vortex transition, vortex breakdown and vortex-turbulence interaction. While a number of these flows are not strictly periodic, it is commonly assumed that the influence of the artificial image flows (in the adjacent boxes) on the flow within the computational domain can be reduced to insignificance by considering a sufficiently large domain size. Most DNS studies use computational domain sizes about 3-10 times larger than the core diameters of the vortices simulated.

The effects of TP periodicity are two-fold. First, the infinite array of periodic image flows exerts on the flow within the computational domain a strain field, whose strength scales inversely as the square of the domain size and which, if sufficiently strong, can trigger the elliptic instability (Kerswell 2002). Second, periodicity implies that the net circulation contained within the domain must be precisely zero. Consequently, an isolated vortex is rendered centrifugally unstable by the Rayleigh criterion. This is seen in figure 1 , where the computationally represented velocity and circulation profiles for an Oseen vortex $\left(\omega_{z}=\omega_{0} \exp \left(-r^{2} / r_{0}^{2}\right)\right.$, where $\omega_{z}$ is the vorticity and $r_{0}$ the core size) are shown for three different computational domain sizes. Even when the computational domain is roughly 30 times the vortex core size $r_{0}$, the region of decreasing circulation $\mathrm{d} \Gamma / \mathrm{d} r<0$ (where $\Gamma=2 \pi r u_{\theta}$ ) is seen to extend up to the core edge. While the negative vorticity associated with this region is weak, the artificial instability of the vortex can exert significant influence on long-term flow evolution. We demonstrate this in the following.

We compare simulations using the classical TP method with those using a spectral algorithm (UB) recently developed by Rennich \& Lele (1997). This scheme is 

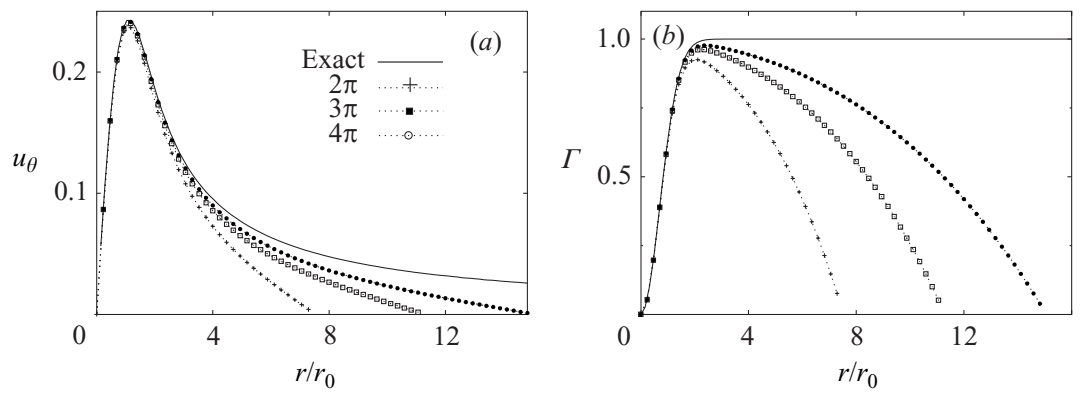

FIGURE 1. Velocity and circulation profiles for an Oseen vortex with core radius $r_{0}=0.13 \pi$ in domain sizes of $2 \pi, 3 \pi$ and $4 \pi$.

well-suited for flows with compact vorticity in domains that are unbounded in two directions and periodic in the third. In UB, the vorticity equation is time-integrated in Fourier space:

$$
\left(\partial / \partial t+\nu k^{2}\right) \widetilde{\omega}(\boldsymbol{k})=-(\mathrm{i} \boldsymbol{k} \times(\widetilde{\boldsymbol{\omega} \times \boldsymbol{u}}))(\boldsymbol{k}),
$$

where $\omega, \boldsymbol{u}$ and $v$ are the vorticity, velocity and kinematic viscosity, respectively; denotes the Fourier transformed field, $f=\sum_{k_{x}, k_{y}, k_{z}} \widetilde{f} \exp (\mathrm{i} \boldsymbol{k} \cdot \boldsymbol{x})$. The vorticity $\boldsymbol{\omega}$ is required to be compact, i.e. $\omega \rightarrow 0$ as $r \rightarrow R$, where $R=X / 2=Y / 2$, and $X$ and $Y$ are the domain sizes in the $x$ - and $y$-directions. The flow is periodic in $z$. The velocity $\boldsymbol{u}$ is decomposed into a rotational component $\widetilde{\boldsymbol{u}}_{\omega}=\mathrm{i} \boldsymbol{k} \times \widetilde{\boldsymbol{\omega}} / k^{2}$ and an irrotational component $\nabla \phi$, which is computed by applying the potential flow boundary condition at $r=R$. The addition of $\nabla \phi$ removes the effects of periodicity in $x$ and $y$. (The profiles for an Oseen vortex in UB coincide with the 'exact' curves in figure 1.) The derivatives of $\boldsymbol{\omega}$ and $\boldsymbol{\omega} \times \boldsymbol{u}$ required for integrating (1) can be computed in Fourier space as these fields are spatially compact. Details of the algorithm are given in Rennich \& Lele (1997).

The TP and UB codes employed here used the third-order compact-storage RungeKutta scheme for time-stepping and the $2 / 3$ truncation rule for dealiasing (Canuto et al. 1988). The UB algorithm requires interpolation of velocity and vorticity fields between Cartesian and cylindrical-polar meshes; herein we employ fourth-order Lagrangian interpolation (shown to be adequate, as discussed later).

Both codes have been well-validated. For instance, the peak value of velocity derivative skewness for decaying homogeneous isotropic turbulence computed using $\mathrm{TP}$ is found to be -0.47 , in good agreement with results in the literature (see e.g. Mansour \& Wray 1994). For UB, we have computed the growth rates of smallamplitude perturbations to the 'q-vortex': $u_{\theta}=q\left(1-\exp \left(-r^{2}\right)\right) / r, u_{z}=\exp \left(-r^{2}\right)$, $u_{r}=0$. In figure 2(a), we plot the evolutions of three-dimensional energy $E$ as predicted by (inviscid) theory (Mayer \& Powell 1992) and as obtained from DNS initialized with a small-amplitude perturbation. Here $q=0.693$, axial wavenumber $k=1.18$, azimuthal wavenumber $m=2$ and the vortex Reynolds number $R e_{\Gamma} \equiv$ circulation/viscosity $=$ $10^{5}$; the simulation uses $96 \times 96 \times 16$ grid points. The growth rates agree within $0.1 \%$.

We first consider the vortex-turbulence interaction problem, a flow which has been studied using DNS (Melander \& Hussain 1993a, hereinafter referred to as $\mathrm{MH}$ ) and analysed via rapid-distortion theory (RDT) (Miyazaki \& Hunt 2000). An Oseen vortex with $r_{0}=0.13 \pi$ is situated within a region of random, fine-scale fluctuations. A homogeneous, isotropic fluctuation field is first generated and then 

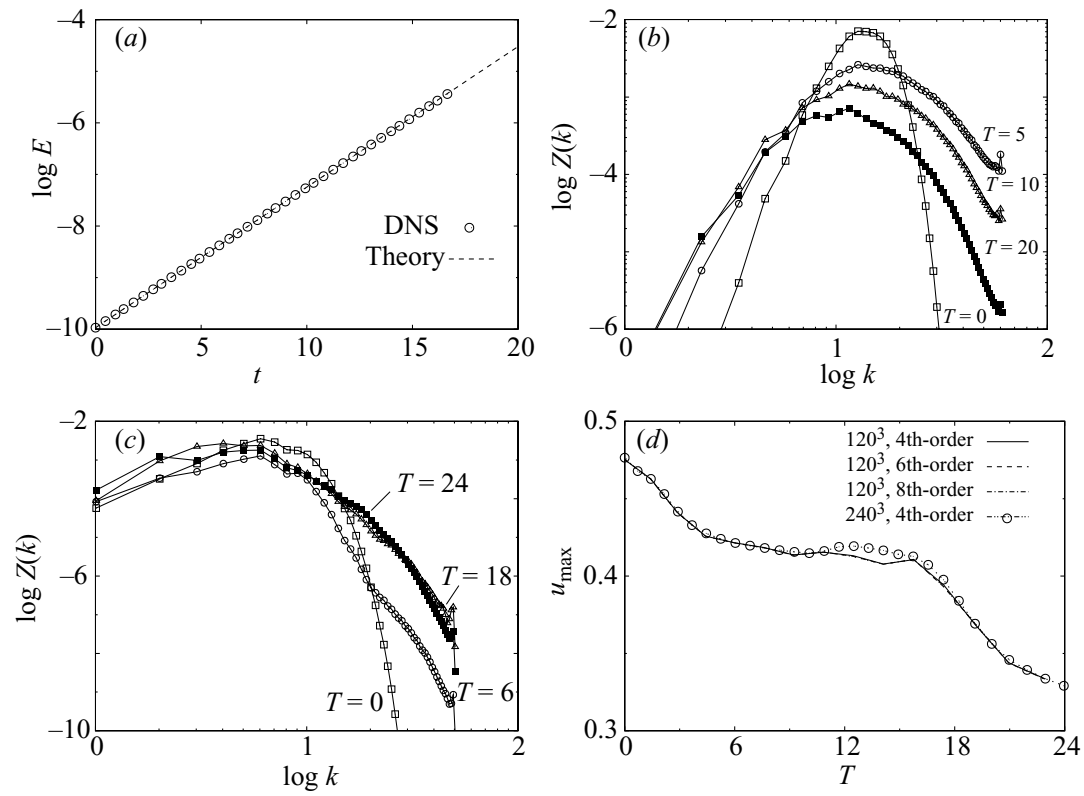

FiguRE 2. (a) Three-dimensional energy $E$ vs. time $t$ for a q-vortex. (b) Spectra of three-dimensional enstrophy $Z(k)$ for UB simulation of vortex-turbulence interaction. (c) Spectra of three-dimensional enstrophy for the UB simulation of $\gamma=0.25$ reconnection. (d) Evolution of domain velocity maxima for UB simulations of $\gamma=0.25$ reconnection.

the 'turbulence' is truncated at a radial extent of $r_{t}=0.95 \pi$ by applying a compact spatial filter $s(r)=\left[\left(\xi^{2}-2\right) / 2\left(\xi^{2}-1\right)\right] \exp \left[\xi^{2} /\left(\xi^{2}-1\right)\right]$, where $\xi=r / r_{t}$. The velocity fluctuations are then rendered divergence-free. The radial truncation to render the turbulent vorticity field compact is unavoidable in UB, but has little effect on the long-time evolution of the flow since turbulence around a vortex decays rapidly when straining is axisymmetric. For instance, RDT analysis shows that the turbulence enstrophy diminishes as $t^{2} / r^{4}$ ( $t$ being time) - even when there is no dissipation of the turbulence via its self-interaction. To confirm the insensitivity of flow evolution to the spatial filtering, we have re-computed the simulation described below: (i) with $r_{t}$ doubled, and (ii) with a spatial filter that eliminates turbulence within the vortex core. In all cases, the turbulence intensities in the vicinity of the core $\left(r<5 r_{0}\right)$ converge to within $10 \%$ of each other after 20 turnover times, and the agreement improves with further evolution. Thus, the quantitative, and certainly the qualitative, aspects of the flow physics observed herein are insensitive to the distinctly different spatial filtering applied. In the simulations discussed below, the initial turbulence intensity $u^{\prime}$ is $10 \%$ of the vortex's peak (azimuthal) velocity. The vortex Reynolds number $\operatorname{Re}_{\Gamma}=6000$ and the flow is simulated using $128^{3}$ grid points in a domain size of $(2 \pi)^{3}$.

Figure 3 compares the TP and UB simulations in terms of vorticity magnitude $\omega$ contours in a meridional plane through the vortex axis. Isovorticity surfaces of flow evolution are shown in figure 4. $T$ denotes time normalized by the vortex turnover time defined in terms of the peak vortex velocity and core radius. Note that the contour increments chosen are identical for the two cases. The initial effect of the vortex on the turbulence is to realign $\omega_{r}$ into $\omega_{\theta}$ and stretch the fine-scale vortex filaments azimuthally. The vortex column's strain first increases with increasing $r$, reaches a peak at the core periphery and then decreases as $1 / r^{2}$. Thus, most of the enstrophy production occurs near the core periphery and this is where the most 

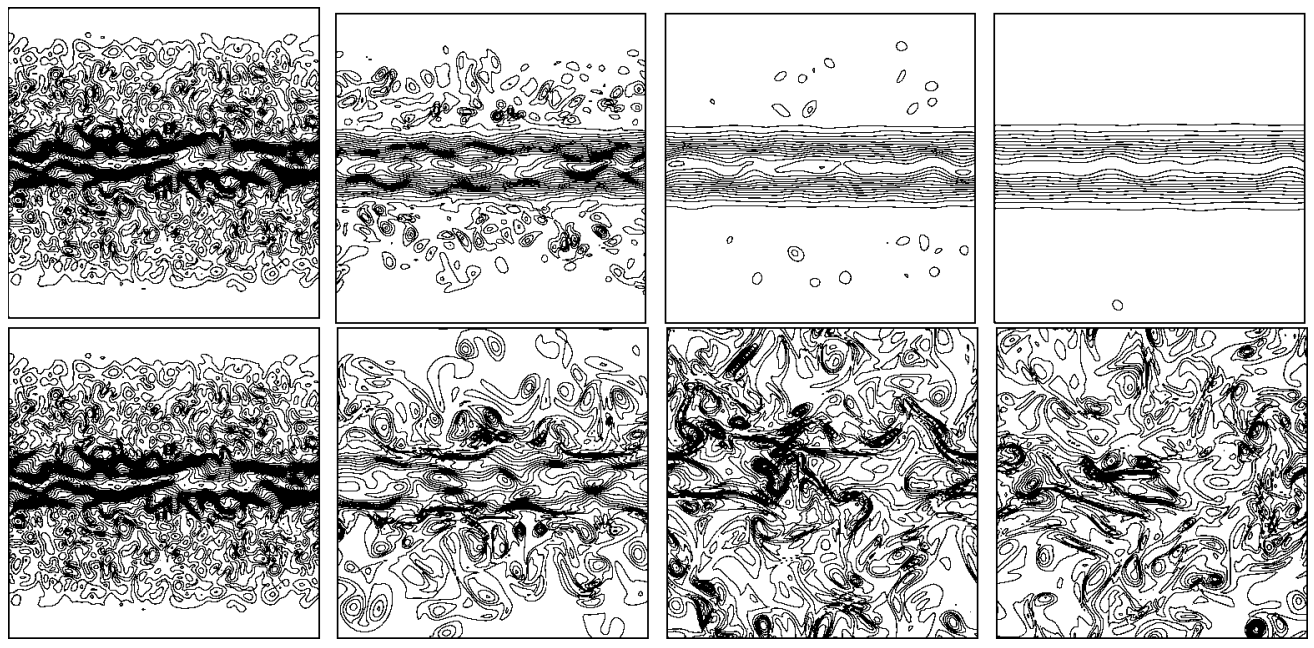

FIgURE 3. Comparison of UB (top row) and TP (bottom row) flow evolutions with identical initial condition. Vorticity contours with increment $0.1 \omega_{0}$ are plotted in a meridional plane. Times (running from left to right in each row) shown are $T=0,30,60$ and 90 for UB and $T=0,60,120$ and 180 for TP.
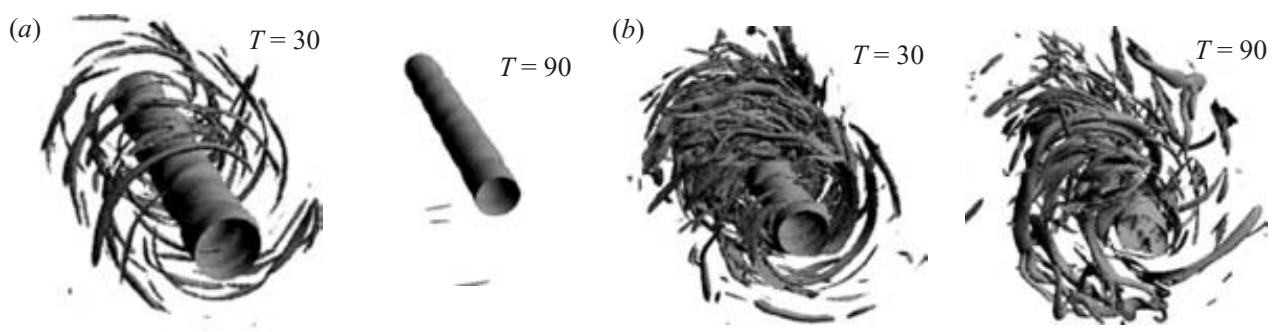

FIgURE 4. Isovorticity surfaces at $T=30\left(\omega=0.3 \omega_{0}\right)$ and $T=90\left(\omega=0.6 \omega_{0}\right)$ for (a) UB and (b) TP.

intense turbulence filaments (vortexlets) are found ( $T=30$ in UB). As the turbulence is organized into ring-like vortexlets wrapping the column $(T=30$ in figure 4$)$, the mutually induced motion of vortexlet dipoles with opposite-signed $\omega_{\theta}$ carries them radially outwards (Pradeep \& Hussain 2003) $(T=60$ in UB) - a process very similar to colliding vortex rings. This process of turbulence reorganization is concomitant with the dissipation-induced decay of the fine scales; at this $R e_{\Gamma}$, dissipation overwhelms production, and hence the turbulence virtually disappears by $T=90$ (UB), leaving behind a laminar vortex column with weak wavy motion in the core (figure 4). In contrast with this monotonic decay of turbulence in UB, turbulence kinetic energy is amplified in TP causing intense vortexlets - found even far away from the vortex column core ( $T=60$ in figure 3 ) where the vortex's strain is very weak. The effect of these vortexlets on the column is two-fold: first, they induce strong bending waves on the vortex column, and, second, the vortexlets peel away the vortex's peripheral vorticity. The latter process is evident at $T=120$ in the form of sheet-like regions of strong vorticity gradients. This process of 'stripping' has been observed in simulations of an array of rings around a vortex column (Marshall 1997). It was found there that stripping requires sufficiently strong rings (having circulation $\sim 20 \%$ of vortex circulation); the absence of stripping in UB is because of rapid turbulence decay. As 

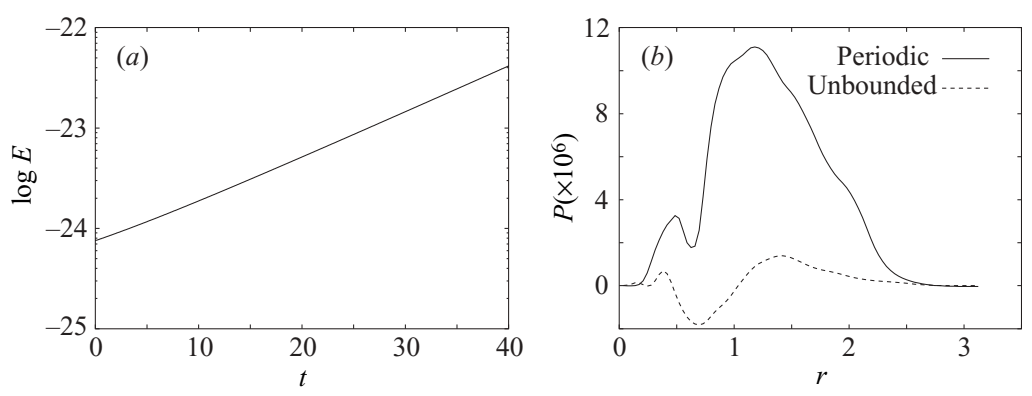

Figure 5. (a) Growth of three-dimensional energy $E$ in TP simulation of an isolated vortex subjected to small-amplitude perturbations; $(b)$ comparison between TP and UB of turbulence production profiles for the simulations in figure 3.

a result of this depletion of vortex-column strength its core undergoes transition into fine-scale turbulence - a process which is complete by $T=180$ (figure 3). Isovorticity surfaces plotted at $T=90$ (figure 4) show the progressive weakening of the vortex core and those at $T=180$ (not shown) confirm the absence of any coherent vortex core.

Standard numerical resolution checks have been performed to ensure that the finescale vortical structures seen in figure 3 are not contaminated by numerical errors. For instance, we plot in figure $2(b)$ the enstrophy spectra for the UB simulation at various times. In the initial period $(0 \leqslant T \leqslant 5)$, a large fraction of three-dimensional energy rapidly cascades to smaller scales. Thus the tail of the enstrophy spectrum lifts up, until about $T=5$, when the dissipation rate is the largest. The fine scales become progressively less energetic with further evolution, and hence the numerical resolution steadily improves. Even at $T=5$, the spectrum has a fall-off of greater than one decade, showing minimal aliasing errors. Similar well-resolved spectra are seen for the corresponding TP simulation. As an additional check on resolution, the UB simulation was re-computed with an increased resolution of $264^{3}$, until $T=25$. The maximum deviation of domain-peak velocity magnitude between the two simulations was less than $1 \%$, confirming that the $128^{3}$ simulation is adequately resolved.

The presence of intense turbulence in the entire computational domain for TP suggests that the simulation is strongly contaminated by the spurious centrifugal instability. To reveal this more clearly, we repeat the TP simulation with the turbulence amplitude reduced to a very low level, so that the simulation well-approximates linear stability analysis. Figure 5(a) plots the growth of three-dimensional energy and shows that the growth is exponential, as is characteristic of a linearly unstable flow. In this limit of small-amplitude perturbations, RDT analysis (Miyazaki \& Hunt 2000) is exact and shows that for a stable vortex three-dimensional energy grows as $t^{2}$ (algebraic growth). The only source of exponential energy growth in this flow is the centrifugal instability due to a velocity profile with $\mathrm{d} \Gamma / \mathrm{d} r<0$ (figure 1).

While the centrifugal instability clearly contaminates the TP simulation (figure 3 ), the artifacts of TP boundary conditions do not occur if $R e_{\Gamma}$ is sufficiently small. To illustrate this, we repeat the simulation of $\mathrm{MH}$ (their case T5) using both $\mathrm{TP}$ and UB methods $\left(\operatorname{Re}_{\Gamma}=665\right)$. In the $\mathrm{MH}$ study, the emphasis being on the evolution of turbulence scales much smaller than the vortex, the external turbulence was initiated with the finest scales permitted by the numerical resolution $\left(128^{3}\right)$. Also, to allow sufficient time for the fine-scale turbulence to survive dissipation, the initial turbulence intensity was very large $(100 \%)$. Consequently, to remove still smaller scales (produced by cascade) than permitted by the resolution, the viscosity had to 
(a)

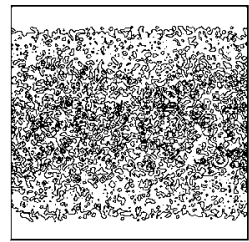

(b)

(c)

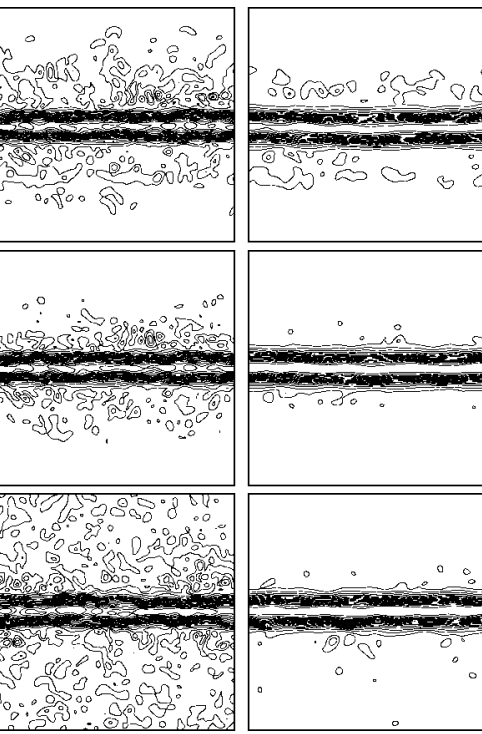

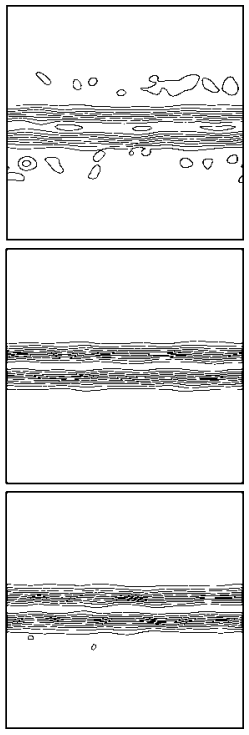

FIGURE 6. $\omega$ contours of UB simulation $(a)$, and TP simulations with spatial filtering $(b)$ and without spatial filtering $(c)$ for MH's case T5. Times (running left to right in each row) are $T=0,4,8,12$ and contour levels $\left[\omega_{\min }, \omega_{\max }, \delta \omega\right]$ are $[4,40,8],[0.25,3.5,0.25],[0.2,2.6,0.2]$, and $[0.2,2.4,0.2]$, respectively.
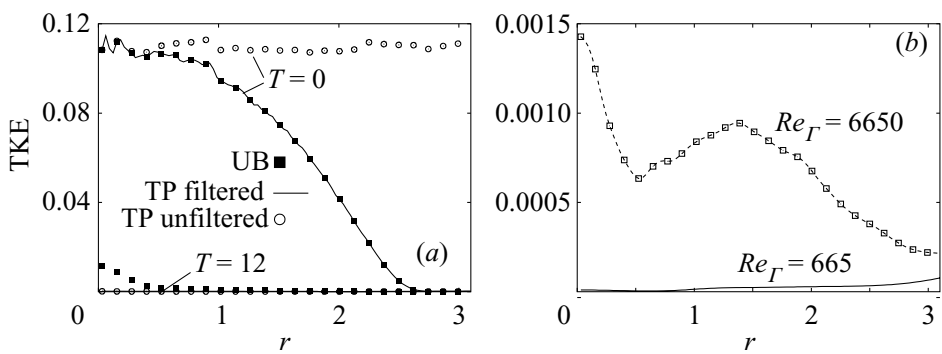

FIGURE 7. Turbulence kinetic energy profiles: $(a)$ UB, TP (with and without spatial filtering) simulations of figure $6 ;(b)$ TP simulations $\left(R e_{\Gamma}=665\right.$ and 6650$)$ at $T=120$.

be chosen high. Thus, $R e_{\Gamma}$ was fairly low in $\mathrm{MH}$. In figure 6 we plot $\omega$ contours for $\mathrm{UB}$ and TP simulations at $R e_{\Gamma}=665$. We perform TP simulations with filtering (for comparison with $\mathrm{UB}$ ) and without filtering (to replicate $\mathrm{MH}$ ). The good agreement between the UB (figure $6 a$ ) and filtered TP (figure $6 b$ ) simulations shows that $R e_{\Gamma}$ here is too low for the centrifugal instability. (This confirms that the results and conclusions of $\mathrm{MH}$ hold.) Note also that the good agreement between the unfiltered TP (figure $6 c$ ) and filtered TP (figure $6 b$ ) cases confirms that spatial filtering of ambient turbulence does not noticeably affect the flow physics (as discussed earlier). In all three cases the turbulence decays monotonically, by viscous damping, and by $T=12$ the vortex is quasi-laminar, as also seen from the profiles of turbulence kinetic energy in figure $7(a)$.

The centrifugal instability effect strengthens as the vortex-core-to-computationaldomain size ratio or as $R e_{\Gamma}$ is increased. Note from figure 1 that the potential for instability exists even for very small core-to-domain size ratios (because $\mathrm{d} \Gamma / \mathrm{d} r<0$ ). At a fixed $R e_{\Gamma}$, the spurious effect strengthens as the core-to-domain size ratio is 


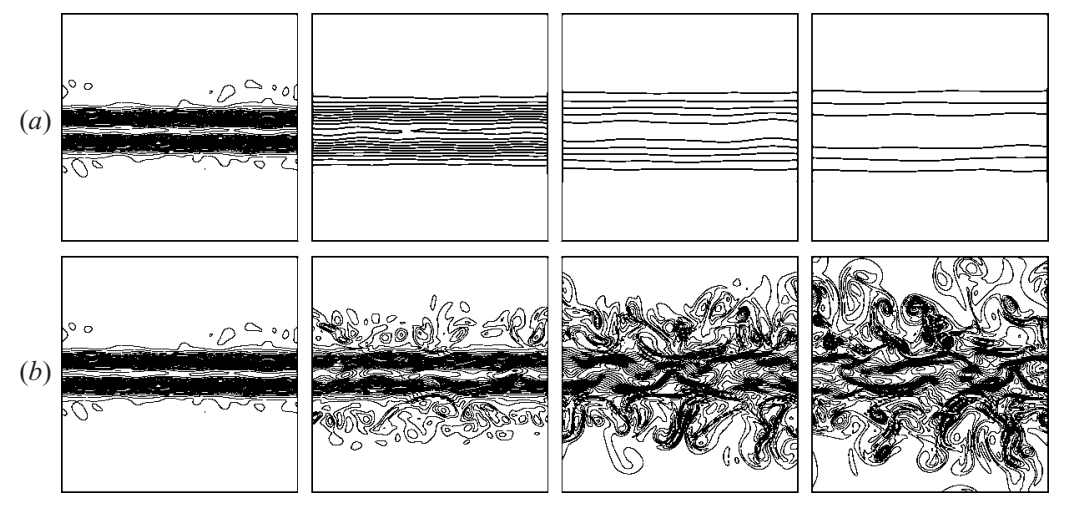

Figure 8. $\omega$ contours for $(a) R e=665$ and $(b) R e=6650$ TP simulations at times (running from left to right in each row) $T=12,40,80,120$. Contour levels are [0.1, 2.5, 0.1].

decreased. And at a given core-to-domain size ratio, the effect strengthens as $R e_{\Gamma}$ is increased. To demonstrate the effect of increasing $R e_{\Gamma}$, we consider the TP simulation of figure 6 with $\operatorname{Re}_{\Gamma}$ increased tenfold. After the initial period of evolution $(0 \leqslant T \leqslant 12)$, the turbulence has sufficiently decayed, permitting an increase of $R e_{\Gamma}$ (by decreasing viscosity) while keeping the numerical resolution the same. Figure 8 compares the evolutions at $\operatorname{Re}_{\Gamma}=665$ and 6650. At the higher $R e_{\Gamma}$, the initially low-amplitude perturbations are amplified most at the core periphery, where $\mathrm{d} \Gamma / \mathrm{d} r<0$. These amplifying perturbations take the form of arrays of vortexlet rings with alternating sign of $\omega_{\theta}$. As the perturbations amplify, they induce largeamplitude wave motion on the core (revealed by application of the helical wave decomposition (see Melander \& Hussain 1993b, for discussion of this technique) in the form of modes that propagate axially with little change in structure) and strip the vortex's peripheral vorticity. By $T=120$, the high- $R e_{\Gamma}$ flow appears fully turbulent a simple consequence of the spurious instability inherent in the boundary condition whereas the low $-R e_{\Gamma}$ flow is laminar, as seen from the profiles of turbulence kinetic energy in figure $7(b)$. We conclude from this comparison that $R e_{\Gamma}$ must be kept small (far smaller than the value permitted by numerical resolution requirements) if errors due to the centrifugal instability effect are to be avoided. This makes the TP method untenable for studying vortex-turbulence interaction, since this interaction is of interest in high-Reynolds-number practical flows.

What is interesting about the centrifugal instability effect in this particular flow is that both the instability and the vortex's strain field promote the formation of ringlike vortexlets around the core. Therefore, TP simulations capture qualitative aspects of flow evolution (at least for short times), but severely overpredict the intensity of the turbulence and the long-time decay of the vortex column. The discrepancy in turbulence quantities between UB and TP evolutions (for the simulations of figure 3) is illustrated in figure $5(b)$. Turbulence production is an order of magnitude larger in TP than in UB. Note that the undulations in UB in figure $5(b)$ disappear when averaged over a large number $(>20)$ of realizations.

As a second illustration of the significance of boundary conditions, we consider a typical case of vortex dynamics: reconnection between two anti-parallel vortices (Kida \& Takaoka 1994). The effect of the zero circulation constraint for TP emerges when the two vortices have unequal strengths (quantified by the circulation ratio $\gamma$ ) a case considered in Marshall, Brancher \& Giovannini (2001) by TP simulations. We consider two columnar vortices with axes aligned along the $z$-direction and centred at 

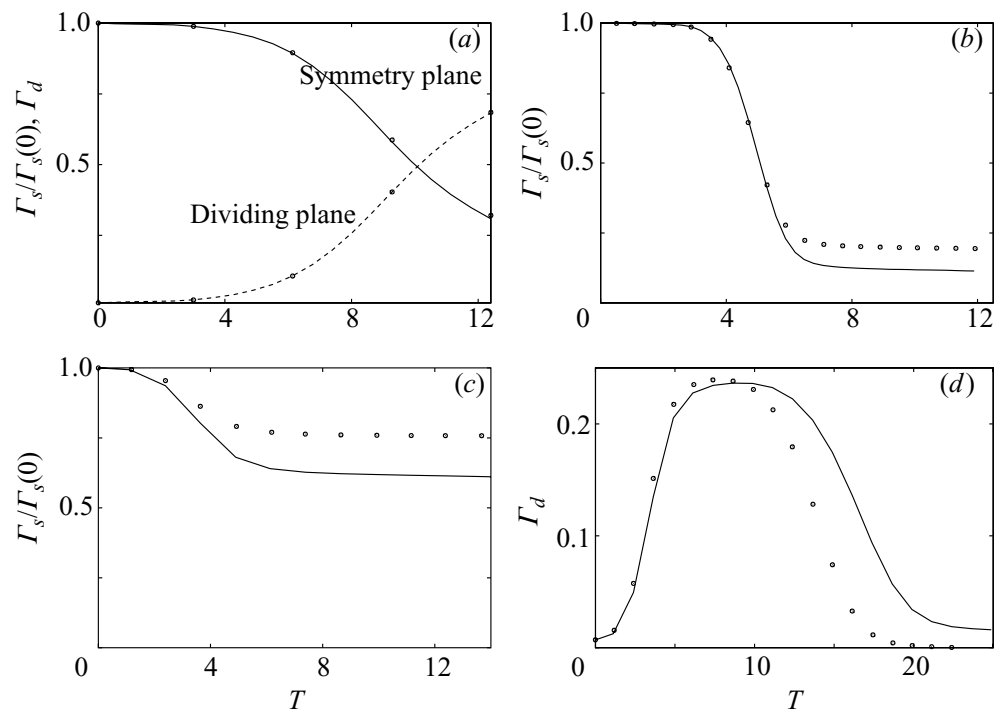

Figure 9. (a) $\Gamma_{s}$ and $\Gamma_{d}$ evolution for $\gamma=1$ reconnection. (b) $\Gamma_{s}$ for $\gamma=0.82$. (c) $\Gamma_{s}$ for $\gamma=0.25$. (d) $\Gamma_{d}$ for $\gamma=0.25$. Lines are TP and symbols UB.

$\left(x_{c}, y_{c}\right)=( \pm \delta, 0)$. The vortices are given a small-amplitude bending wave perturbation such that their self-induced motions cause them to move towards each other. The rate at which reconnection proceeds can be quantified by measuring the circulations $\left(\Gamma_{s}\right.$ and $\left.\Gamma_{d}\right)$ in the 'symmetry' $(z=0)$ and 'dividing' $(x=0)$ (half-) planes. Note that the vortex cores are initially closest in the symmetry plane. The dividing plane has special significance in the $\gamma=1$ case, being the plane in which $\omega_{z}$ is identically zero at all times. The circulations $\Gamma_{d}$ and $\Gamma_{s}$ are defined as

$$
\Gamma_{s}=\int_{y=-Y / 2}^{Y / 2} \int_{x=-X / 2}^{0} \omega_{z}(x, y, 0) \mathrm{d} x \mathrm{~d} y, \quad \Gamma_{d}=\int_{y=-Y / 2}^{Y / 2} \int_{z=0}^{Z / 2} \omega_{x}(0, y, z) \mathrm{d} z \mathrm{~d} y,
$$

where $X, Y$ and $Z$ are the domain dimensions. In the simulations discussed below the vortex cores are 1/30th the domain size, and the simulations are performed at $\operatorname{Re}_{\Gamma}=1500$ using $120^{3}$ grid points (these flow parameters are identical to those of Marshall et al. 2001).

First, consider the two vortices with equal circulations $(\gamma=1)$. As reconnection proceeds, circulation is transferred from the symmetry plane to the dividing plane such that $\Gamma_{d}+\Gamma_{s}=$ constant, as required by the divergence-free nature of vorticity. It is well-known that reconnection does not proceed to completion, i.e. $\Gamma_{s}$ does not tend to zero at large times but instead asymptotes to a finite value. Remnants of the original vortex core ('threads') remain with vorticity along $z$. An important theoretical problem is to predict thread circulation as a fraction of the original vortex circulation, i.e. $\Gamma_{s}(t) / \Gamma_{s}(0)$. In figure $9(a-c)$, we plot this quantity for three cases: $\gamma=1,0.82$ and 0.25 . For $\gamma=1$, the net circulation in the flow is zero. Consequently, TP and UB simulations agree, as expected. However, there are significant discrepancies between the two methods when $\gamma \neq 1$. The simulations disagree by roughly $30 \%$ for $\gamma=0.82$ and by $25 \%$ for $\gamma=0.25$. The difference arises because the TP method is unable to accurately represent the azimuthal velocity profiles and hence the true value of $\Gamma_{s}(t)$ because of the zero domain circulation constraint. Thus the quantitative predictions of the degree of reconnection completeness obtained from TP are erroneous. 

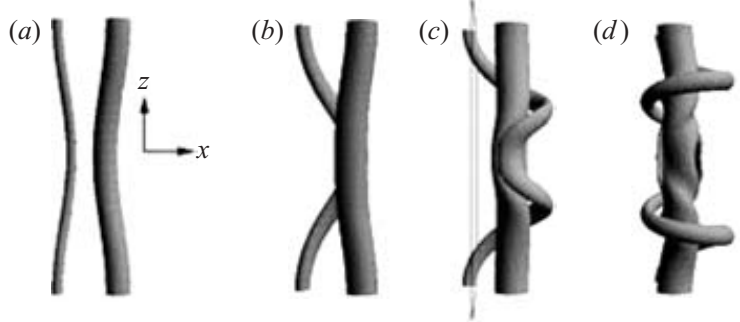

FiguRE 10. Isovorticity surfaces $(\omega=0.2)$ at $T=0,6,12$ and $18(a-d)$ for UB simulation of interaction between two anti-parallel vortices of unequal strength. Dividing plane $(x=0)$ shown in $(c)$.

Insignificant errors in all the reconnection cases have been confirmed by checking that there is no 'pile-up' in the enstrophy spectra. For instance, we plot the spectra for the UB $\gamma=0.25$ reconnection simulation in figure $2(c)$. The spectra decay by more than two decades at all times, showing the aliasing errors to be insignificant. Spectra in TP simulations are equally well-resolved. As an additional check, we compare in figure $2(d)$ the evolution of domain-peak velocities for $\gamma=0.25$ reconnection computed using $120^{3}$ and $240^{3}$ grid points. The maximum deviation between the two is less than $2 \%$. The interpolation of velocity and vorticity fields between Cartesian and cylindrical-polar meshes required in UB is a potential source of loss of numerical accuracy. Figure $2(d)$ also plots the evolution of domain-peak velocity magnitude obtained using fourth-, sixth- and eighth-order Lagrangian interpolations, all $120^{3}$. These curves are visually indistinguishable. Thus, $120^{3}$ fourth-order simulations are adequate. These checks show that the quantitative differences seen between TP and UB simulations are an artifact of boundary conditions, and not numerical errors.

While TP and UB simulations broadly agree on the qualitative aspects of the vortex dynamics for $\gamma \neq 1$, there are some subtle differences between the two. We focus on the $\gamma=0.25$ case. Flow evolution in the UB simulation is illustrated in terms of isovorticity surfaces in figure 10. The main physical mechanism in this flow is the wrapping of the weaker vortex around the stronger column with its ring-like legs self-advecting along the column. Continued evolution of this flow at large $R e$ would result in a hierarchy of 'rings', which induce bending and axial waves in the column's core. TP and UB simulations agree well in these qualitative aspects, but there are quantitative differences in the rate of wrapping of the weaker vortex around the stronger column. This is indicated by the difference in $\Gamma_{d}$ evolutions in figure $9(d)$. Initially, the only contribution to $\Gamma_{d}$ comes from the slight bending wave perturbation given to the vortices ( $T=0$ in figure 10). As the weaker vortex is wrapped, its vorticity is tilted further into the $x$-direction and hence $\Gamma_{d}$ increases. As the wrapped vortex is wound closer to the column, it induces strong core dynamics on the column (Marshall et al. 2001), generating $\omega_{x}$ in the column's core. Core dynamics-induced $\omega_{x}$ in the column is of opposite sign to the $\omega_{x}$ of the weaker vortex. This effect is illustrated in figure $11(a)$, where $\omega_{x}$ occurs in two dipole-like patches, where one patch is the leg of the weaker vortex and the opposite-signed patch is $\omega_{x}$ induced within the stronger vortex. These regions have nearly equal circulations by $T=18$ and hence $\Gamma_{d}$ becomes nearly zero in the UB case. Such patches are also seen in TP, but with stronger $\left|\omega_{x}\right|$ (figure 11b). More significantly, however, the weaker vortex is swept around the column more slowly in TP. This is indicated by the presence of the two lowest cells of $\omega_{x}$ in the $(y, z)$-plane. These cells are cross-sections of the legs of the weaker 

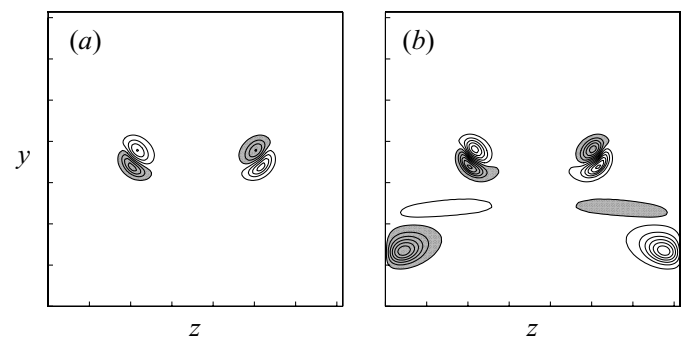

FIGURE 11. $\omega_{x}$ contours at $T=18$ in the $x=0$ plane (shown in figure $10 c$ ) for $(a)$ UB and $(b)$ TP cases. Shaded regions contain $-\omega_{x}$. Contour increment $\delta \omega_{x}=0.02$.

vortex and do not appear in UB because the weaker vortex no longer intercepts the dividing plane. The persistence of these $\omega_{x}$ cells in TP causes the slower decay of $\Gamma_{d}$ (figure $9 d$ ).

These differences arising from different boundary conditions are remarkable because the vortex cores are a very small fraction of the domain size. Thus, the effect of straining by the image flows is negligible. Yet, as we show, periodicity can significantly contaminate quantitative predictions, such as the reconnection time scale - which depends on the delicate balance between vortex stretching, tilting and diffusion.

In summary, the results here emphasize that TP simulations must be avoided whenever the net circulation of the flow being simulated is non-zero - even if the vortices are small compared to the domain size. When the net circulation in the flow is zero, the TP method may well be preferable as it requires about half the computational time. Rennich \& Lele (1997) present evidence that even for zero net circulation flows the UB method is superior because the domain size can be much smaller than in TP, but this advantage may not translate to all flows. An example is trailing vortex decay in ambient turbulence. For non-zero circulation flows, it is clearly preferable to use more advanced schemes than TP, such as the Rennich \& Lele algorithm.

\section{REFERENCES}

Canuto, C., Hussaini, M. Y., Quarteroni, A. \& Zang, T. A. 1988 Spectral Methods in Fluid Dynamics. Springer.

Kerswell, R. R. 2002 Elliptical instability. Annu. Rev. Fluid Mech. 34, 83.

Kida, S. \& Takaoka, M. 1994 Vortex reconnection. Annu. Rev. Fluid Mech. 26, 169.

Mansour, N. N. \& Wray, A. A. 1994 Decay of isotropic turbulence at low Reynolds number. Phys. Fluids 6, 808 .

Marshall, J. S. 1997 The flow induced by periodic vortex rings wrapped around a columnar vortex core. J. Fluid Mech. 345, 1.

Marshall, J. S., Brancher, P. \& Giovannini, A. 2001 Interaction of unequal anti-parallel vortex tubes. J. Fluid Mech. 446, 229.

Mayer, E. W. \& Powell, K. G. 1992 Viscous and inviscid instabilities of a trailing vortex. J. Fluid Mech. 245, 91.

Melander, M. V. \& Hussain, F. 1993a Coupling between a coherent structure and fine-scale turbulence. Phys. Rev. E 48, 2669 (referred to herein as MH).

Melander, M. V. \& Hussain, F. 1993 b Polarized vortex dynamics on a vortex column. Phys. Fluids A 5, 1992.

MiYAzAKI, T. \& HunT, J. C. R. 2000 Linear and nonlinear interactions between a columnar vortex and external turbulence. J. Fluid Mech. 402, 349.

PradeEP, D. S. \& Hussain, F. 2003 Interaction between a coherent structure and turbulence. In Advances in Fluid Mechanics (ed. M. Alam et al.), p. 272. JNCASR, Bangalore, India.

Rennich, S. C. \& Lele, S. K. 1997 Numerical method for incompressible vortical flows with two unbounded directions. J. Comput. Phys. 137, 101. 